\title{
El perfil docente en bibliotecología en la Escuela Nacional de Biblioteconomía y Archivonomía (México)
}

\author{
The Teaching Profile at the National School of \\ Library Science and Archive Science
}

\begin{abstract}
Dra. Brenda Cabral Vargas * Instituto de Investigaciones Bibliotecológicas y de la Información, UNAM
\end{abstract}

\begin{abstract}
Resumen
El objetivo de este documento es describir la formación y actualización continua de los docentes de la Escuela Nacional de Biblioteconomía y Archivonomía(ENBA). Los resultados fueron obtenidos a través de un estudio de campo, utilizando un cuestionario como herramienta de recopilación de la información.No se pretende describir un listado de cursos o eventos académicos en los que han participado los docentes en esta institución, sino que se intenta dilucidar qué elementos cognitivos, actitudinales, valorativos y de interacción con los alumnos está favoreciendo el proceso de enseñanza aprendizaje en las diferentes instituciones educativas a nivel hispanoamericano, en el ámbito bibliotecológico; en mi caso expondré la situación de los docentes en Biblioteconomía de la ENBA que pertenece a la Secretaría de Educación Pública (México), pero que, a partir de mediados del 2017, está en proceso de incorporación al Instituto Politécnico Nacional del mismo país.
\end{abstract}

Palabras clave:Perfil docente, México, Biblioteconomía, Formación docente. 


\begin{abstract}
The purpose of this document is to describe the training and continual updating of teachers at the Escuela Nacional de Biblioteconomía y Archivonomía(ENBA), It was conducted a study of field, using as a tool for gathering the information a questionnaire, where the results are not intended to describe a list of courses or academic events in those who have been involved teachers in this institution, but, itis trying to elucidate what elements cognitive, attitudinal, evaluative and interaction with students is favoring the teaching and learning process in the different educational institutions at the Hispanic American level in the librarian field. In this case will be discussed the situation of teachers in Library Science in the ENBA, which belongs to the SEP, who are in these moments in the process of incorporation into the InstitutoPolitecnico Nacional in Mexico.
\end{abstract}

Keywords: Teacher profile, Mexico, Library Science, Teacher, Training.

\title{
I. Introducción
}

El perfil profesional del docente, si lo consideramos desde el punto de vista de las funciones, está cambiando a pasos agigantados, debido a que se requiere de otros conocimientos, aptitudes y habilidades adicionales al dominio del área de estudios que se imparte y, por tanto, se tiene que pasar a un nuevo perfil o a un perfil con adaptaciones. En el momento actual, el profesor requiere de nuevas estrategias didácticas, percepciones, experiencias y conocimientos para dar respuesta a las múltiples demandas por parte de las instituciones educativas, pero además a los intereses de sus propios alumnos.

Como expresa Borrero (2012) "la relevancia del rol que debe desempeñar el profesor en el ámbito educativo de nivel universitario, un tema muy controvertido en la actualidad y sometido a revisión y cambio constante para la adaptación al nuevo Espacio Europeo de Ecuación Superior" (p.237).Si vemos, no es algo fácil ya que como bien menciona la autora es controvertido y está en constante cambio. También, de este perfil llama la atención que no 
solo debe venir lo que las autoridades crean o digan, sino que la opinión de los estudiantes debe ser tomada en cuenta. Al respecto la autora cita que:

El objetivo es conocer la opinión del alumnado sobre lo que considera que es un buen docente, que debe ir más allá de las encuestas de calidad planteadas por muchas Universidades. Los/as alumnos/as deben y pueden ofrecer criterios de valoración sobre el perfil docente y el rol desempeñado como profesionales de la docencia a nivel universitario más allá de los cuestionarios cerrados existentes. (Borrero, 2012, p.238)

Debemos también reflexionar en torno a que las universidades están sufriendo muchísimas transformaciones debido a la globalización y los cambios tecnológicos, lo que provoca que estén viviendo una de las mayores transformaciones y retos a los cuales nunca antes se habían enfrentado.

Como cita Mas (2012) que:

Para abordar satisfactoriamente estas nuevas necesidades formativas, para establecer referentes adecuados en los protocolos de acreditación, selección, promoción y formación del profesorado universitario, debe delimitarse el nuevo perfil competencial que debe atesorar el mencionado profesional para desarrollar convenientemente sus funciones docente, investigadora y gestora, considerando sus diferentes escenarios de actuación profesional”. (p.299)

En todas las disciplinas el cambio en los perfiles docentes es un requerimiento insoslayable y que se requiere para mejorar el nivel de calidad de la educación tanto a nivel nacional como internacional.

\section{Concepto de perfil docente}

En la investigación de áreas educativas, el papel del profesor o docente cada vez se presenta más como un guía del aprendizaje del alumno que como un medio transmisor de conocimientos. Así, en diferentes partes del mundo, se ha trabajado en modelos de docencia 
que integran las características académicas y sociales ideales de cada profesor. Estos rasgos constituyen el perfil docente, término que, de forma más concreta, comprende los conocimientos, habilidades y actitudes para generar experiencias de aprendizaje y desarrollar competencias genéricas en los estudiantes. Cabe indicar que cada docente tendrá un perfil amoldado a sus cualidades individuales de carácter ético, social y académico.

Para entender apropiadamente el perfil docente - específicamente en la educación superior Mas (2012) recomendó identificar los niveles de actuación que tiene el docente. De manera que, como primer escenario, el docente se desempaña a nivel general, es decir, en un entorno social laboral, de cultura y profesional; en segundo lugar, forma parte de la institución, pues está adscrito a un departamento, facultad o universidad. Finalmente tiene incidencia dentro de su propia aula, laboratorio o seminario. Con base en lo anterior, el autor propone seis competencias que integrarían el perfil docente universitario:

- Diseñar la guía docente de acuerdo con las necesidades, el contexto y el perfil profesional, todo ello en coordinación con otros profesionales.

- Desarrollar el proceso de enseñanza y aprendizaje propiciando oportunidades de aprendizaje tanto individual como grupal.

- Tutorizar el proceso de aprendizaje del alumno propiciando acciones que le permitan una mayor autonomía.

- Evaluar el proceso de enseñanza y aprendizaje.

- Contribuir activamente a la mejora de la docencia.

- Participar activamente en la dinámica académico-organizativa de la institución.

A partir del perfil que nos propuso Mas (2012) queda claro que el docente debe estar preparado para actuar frente a un grupo, pero además, se necesita de su disposición para tomar partido en el desarrollo y crecimiento mismo de la institución en donde labora. De lo anterior, también reflexionamos que se deben impulsar en las universidades las condiciones propicias para que el docente sepa cuán importante puede ser su participación en la mejor de la educación impartida; se observa como reto conseguir que los propios docentes se contemplen como agentes de la difusión de la cultura e investigación -además de la 
docencia- solo así se lograría consolidar un perfil apto para el cambio positivo en las universidades.

Además de lo expuesto con anterioridad respecto al perfil docente, podemos aproximarnos un poco más al concepto, al examinar por separado las palabras que componen el término. Así tenemos que para Sánchez (2005) un perfil integra las "aptitudes, destrezas y conocimiento que debe poseer un individuo para desempeñar una tarea específica" (p. 305). En los puestos de trabajo, la misma fuente citada sugiere que el perfil describe los requisitos que se deben cubrir para optar por la vacante. Como observamos, en la definición de perfil no se habla, necesariamente, de labores profesionales, así que en el Glosario de educación superior publicado en 1998, se incluyó el término perfil profesional, que engloba los requisitos para ejercer una profesión. Estos se agrupan en:

- Psicológicos: capacidades, habilidades y aptitudes.

- Técnico-científicos: conocimientos y destrezas.

- Sociales: relaciones humanas y legislación.

- Culturales: información, etc.

Por su parte, el docente es, según Perrone (2007), un profesional que guía el aprendizaje del estudiante en cierto nivel educativo, así que su preparación se da en escuelas normales o en las universidades. Perrone (2007) también sostiene que la labor del docente es "formar ciudadanos responsables, críticos, autónomos y reflexivos" (p.147). Es, por tanto, un eje central de la vida en sociedad, así que Perrone (2007) considera necesaria su capacitación constante y compromiso con la labor, la cual debiera ser recompensada con un salario digno de un profesional. El Cuadro 1 muestra las expectativas actuales de los procesos de enseñanza y aprendizaje versus a cómo eran entendidos en otro momento. Como se percibe en el Cuadro 1 ha cambiado de manera considerable lo que se esperaba que sucediera dentro del proceso de enseñanza y aprendizaje a través del tiempo. 


\section{Cuadro 1}

Expectativas actuales de los procesos de enseñanza y aprendizaje

\begin{tabular}{|c|c|c|}
\hline Aspectos & Ahora & Antes \\
\hline Aprendizaje & $\begin{array}{l}\text { - Construcción activa } \\
\text { - Conexiones } \\
\text { - Situado }\end{array}$ & $\begin{array}{l}\text { - De la información } \\
\text { - Jerárquico } \\
\text { - Descontextualizado }\end{array}$ \\
\hline Enseñanza & $\begin{array}{l}\text { - Transformación } \\
\text { - Andamiaje }\end{array}$ & $\begin{array}{l}\text { - Transmisión } \\
\text { - Directo }\end{array}$ \\
\hline Currículum & - Maleable & - Fijo \\
\hline Tareas & $\begin{array}{l}\text { - Auténticas } \\
\text { - Conjunto de representaciones }\end{array}$ & $\begin{array}{l}\text { - Aisladas } \\
\text { - Materiales secuenciales }\end{array}$ \\
\hline Mediación social & $\begin{array}{l}\text { - Comunidad de aprendizaje } \\
\text { - Colaboración } \\
\text { - Discurso }\end{array}$ & $\begin{array}{l}\text { - Individual } \\
\text { - Competición } \\
\text { - Recitación }\end{array}$ \\
\hline Herramientas & $\begin{array}{l}\text { - Uso interactivo de la } \\
\text { computadora }\end{array}$ & - Papel y lápiz \\
\hline Evaluación & $\begin{array}{l}\text { - Basado en actuaciones } \\
\text { - Carpeta individual }\end{array}$ & $\begin{array}{l}\text { - Pruebas de rendimiento } \\
\text { - Test estandarizados }\end{array}$ \\
\hline
\end{tabular}

Fuente: Cano, E. (2005). El portafoliodel profesorado universitario: un instrumento para la evaluación y el desarrollo profesional. Barcelona: Universitat de Barcelona, Institut de Ciències de l'Educació.

\section{Situación en México}

En México la institución que regula los asuntos en materia de educación superior es la Subsecretaría de Educación Superior, órgano dependiente de la Secretaría de Educación Pública (SEP). Hasta el momento, no se ha podido localizar un programa que establezca la naturaleza del perfil docente en la universidad.

La referencia más cercana es el Programa Sectorial de Educación 2013-2018, documento en el que, de manera recurrente, se enfatiza la necesidad de dotar a los docentes, además de los directivos y supervisores de la educación superior, con los conocimientos y herramientas para propiciar la permanencia en este nivel. Se otorga también un peso relevante al uso de 
tecnologías de la información y comunicación y el impulso a las carreras de ciencia y tecnología, sin pretender el olvido de una educación integral inmersa en la cultura y los valores.

Por lo anterior, para este estudio se tomaron como fundamento algunas estipulaciones de la obra de la Secretaría de Educación Pública (SEP) (2014) titulada Perfiles, Parámetros e indicadores, el cual fue elaborado para la educación media superior, por lo cual se considera apropiado, pues es el nivel que le antecede al superior. En tal documento se establece que la conformación de un perfil docente:

Permitirá conocer no sólo las características de ingreso de los docentes y técnico docentes en la educación superior, sino también las necesidades de formación y actualización docente, lo que incidirá favorablemente en el reforzamiento del conocimiento, así como la necesidad de una formación continua por parte de los profesores, que mantenga al nivel educativo en un proceso permanente de actualización y pertinencia de los conocimientos que se imparten. (SEP, 2014, p. 1)

Así, en el Cuadro 2 se detalla el perfil del docente según el SEP.

\section{Cuadro 2}

\section{Dimensiones del Perfil Docente del SEP}

\begin{tabular}{|c|l|}
\hline Dimensión & \multicolumn{1}{|c|}{ Aspecto } \\
\hline $\mathbf{1}$ & $\begin{array}{l}\text { Domina y estructura los saberes para facilitar experiencias de aprendizaje } \\
\text { significativo. }\end{array}$ \\
\hline $\mathbf{2}$ & $\begin{array}{l}\text { Planifica los procesos de enseñanza y de aprendizaje atendiendo al enfoque } \\
\text { por competencias y los ubica en los contextos disciplinares, curriculares y } \\
\text { sociales amplios. }\end{array}$ \\
\hline $\mathbf{3}$ & Evalúa los procesos de enseñanza y aprendizaje con un enfoque formativo. \\
\hline $\mathbf{4}$ & $\begin{array}{l}\text { Organiza su formación continua a lo largo de su trayectoria profesional. } \\
\text { Lleva a la práctica procesos de enseñanza y aprendizaje de manera efectiva, } \\
\text { creativa e innovadora en su contexto institucional. }\end{array}$ \\
\hline
\end{tabular}

Fuente: Secretaría de Educación Pública (2014). Perfil, parámetros e indicadores para el ingreso a las funciones docentes y técnico docentes en la educación media superior. 


\section{a. El perfil docente en bibliotecología}

La presente investigación parte del siguiente problema identificado: Los estudios realizados sobre el perfil del docente y sobre los modelos educativos se han venido desarrollando de manera independiente; sin embargo, mucho se ha insistido en que la educación superior debe contribuir a la formación de profesionales con sentido crítico, creativo y emprendedor para enfrentar los retos de las sociedades de la información y del conocimiento y para lograrlo, uno de los elementos clave es el docente ya que es él quien será el eje para que se lleve a cabo cualquier plan de estudios.

\section{Desarrollo}

De tal forma, la presente investigación parte de las siguientes preguntas:

$\checkmark$ ¿Cuál es el conjunto de conocimientos, habilidades y aptitudes de los docentes de la Escuela de Bibliotecología y Archivonomía?

$\checkmark$ ¿Cuáles son las estrategias institucionales que se han implementado para que el perfil del docente sea congruente con el modelo educativo de los planes de estudio de las escuelas de bibliotecología y documentación en Iberoamérica y el Caribe?

En consonancia con lo expuesto en apartados anteriores, surgió el interés por conocer el nivel de dimensiones que cubren del perfil, mostrado en el cuadro anterior, los profesores que se desempeñan en la Escuela Nacional de Biblioteconomía y Archivonomía (ENBA). El trabajo de campo que se realizó mediante la aplicación del instrumento de recolección de datos dirigido a docentes de la ENBA a partir de lo cual se generaron resultados en cuanto a la identificación del perfil de cada docente para, posteriormente, realizar el análisis y proporcionar los resultados de la investigación. Se aplicó un cuestionario con preguntas abiertas y cerradas a profesores de la modalidad presencial y a distancia, lamentablemente, solo se contó con el apoyo de 14 de ellos. Los resultados se muestran en el Gráfico 1 y 2. 


\section{Gráfico 1}

Género de los participantes

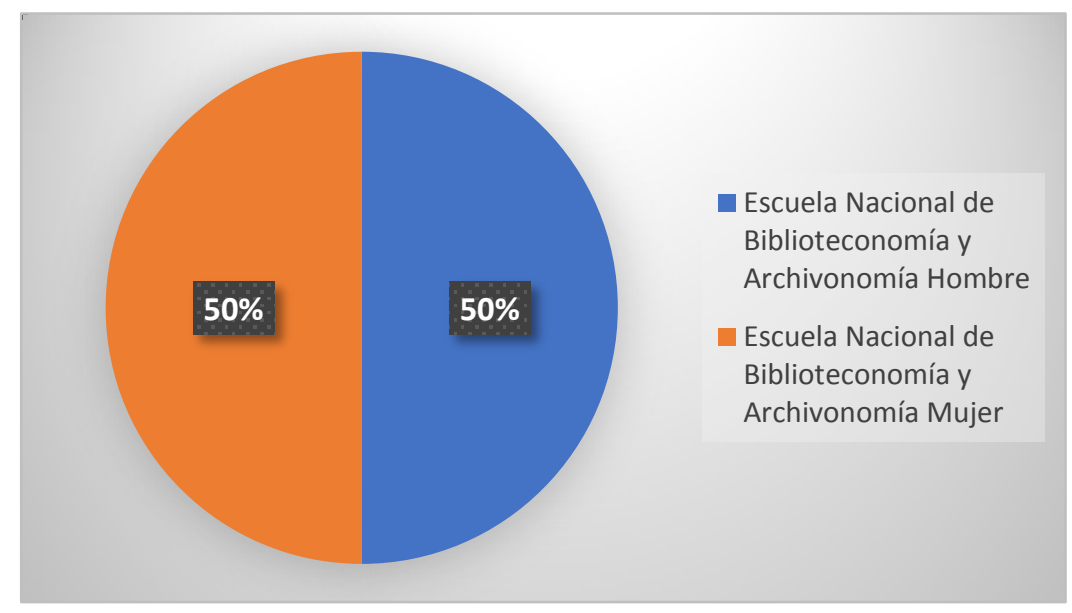

Fuente: elaboración propia de la autora (2017).

Una participación equitativa entre el profesorado de la ENBA, es decir, 12 fueron docentes del sexo masculino y 12 del femenino.

\section{Gráfico 2}

\section{Edad de los participantes}

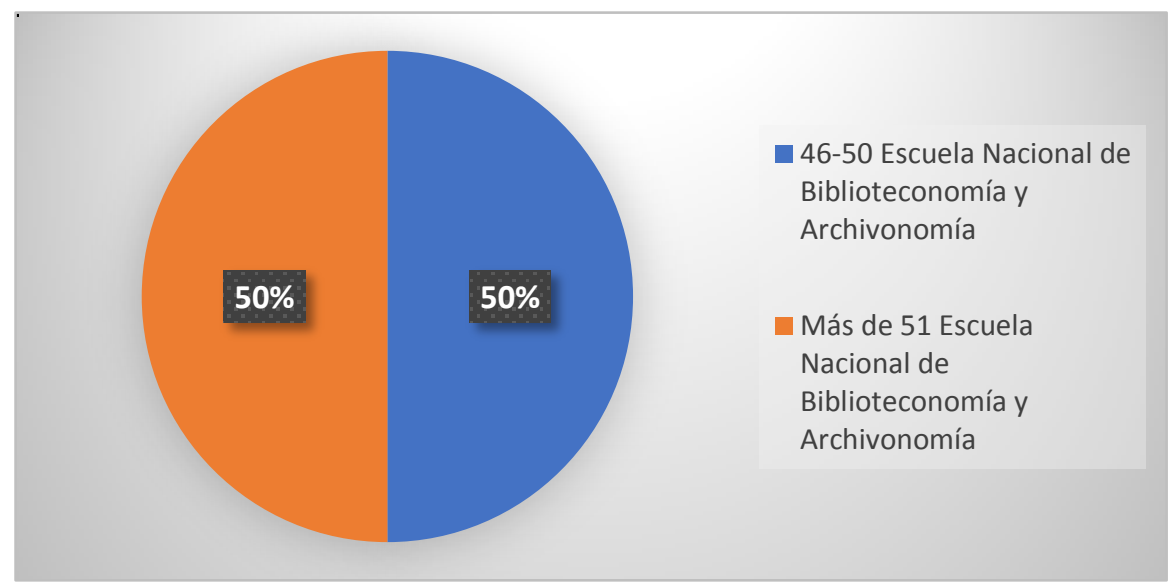

Fuente: elaboración propia de la autora (2017).

La edad del profesorado se distribuye por igual (50\%), en rangos que van de los 46 a los 50 años y más de 51 años. De estos participantes el $75 \%$ cuenta con licenciatura y solo $25 \%$ con posgrado. Además, se identificó que: 
- La mitad de los profesores son personal contratado por asignatura u hora/clase, la otra mitad se dedica exclusivamente al ejercicio docente.

- La antigüedad de los académicos que imparten docencia va en proporción con un $25 \%$, repartido en años que oscilan desde hace 10, 15, 17 y 20 años.

- De igual manera, la formación académica de profesores tuvo una distribución del 25\% para los rubros de licenciatura en comunicación, licenciatura en biblioteconomía, licenciatura en biblioteconomía con maestría en gestión de la información y pasante de la maestría en administración

- Una mitad de los participantes dijo tener de 16 a 20 años de experiencia docente, la otra parte se distribuye en porciones del $25 \%$ con experiencia docente de 21 a 25 años y con más 26.

- La gran mayoría de los docentes han tomado mínimamente algún curso en sus líneas docentes (Gráfico 3).

\section{Gráfico 3}

\section{Áreas de los cursos tomados}

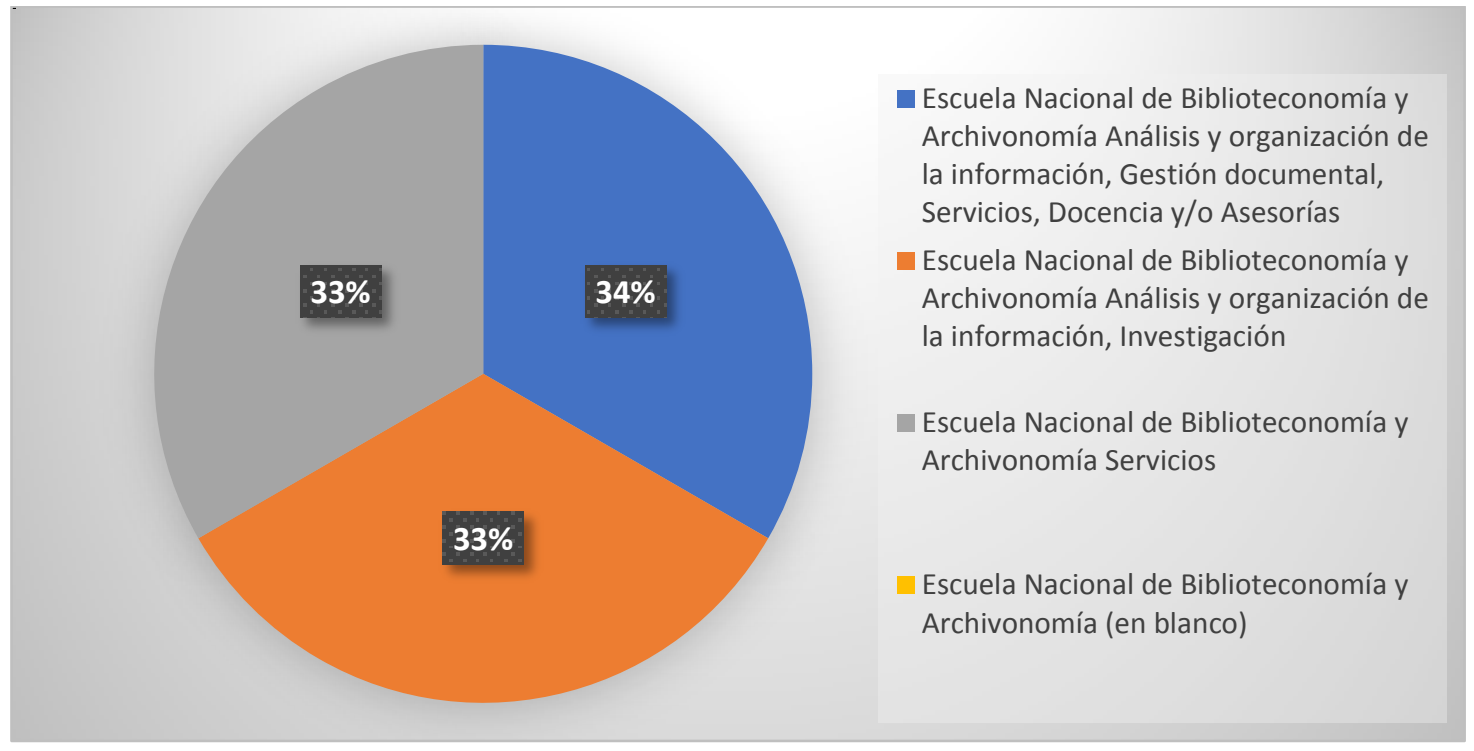

Fuente: elaboración propia de la autora (2017).

El área de estudios al que pertenecen las asignaturas impartidas por los profesores durante los últimos tres años se distribuyen en bloques del $25 \%$. 


\section{Gráfico 4}

Materias impartidas en los últimos 3 años

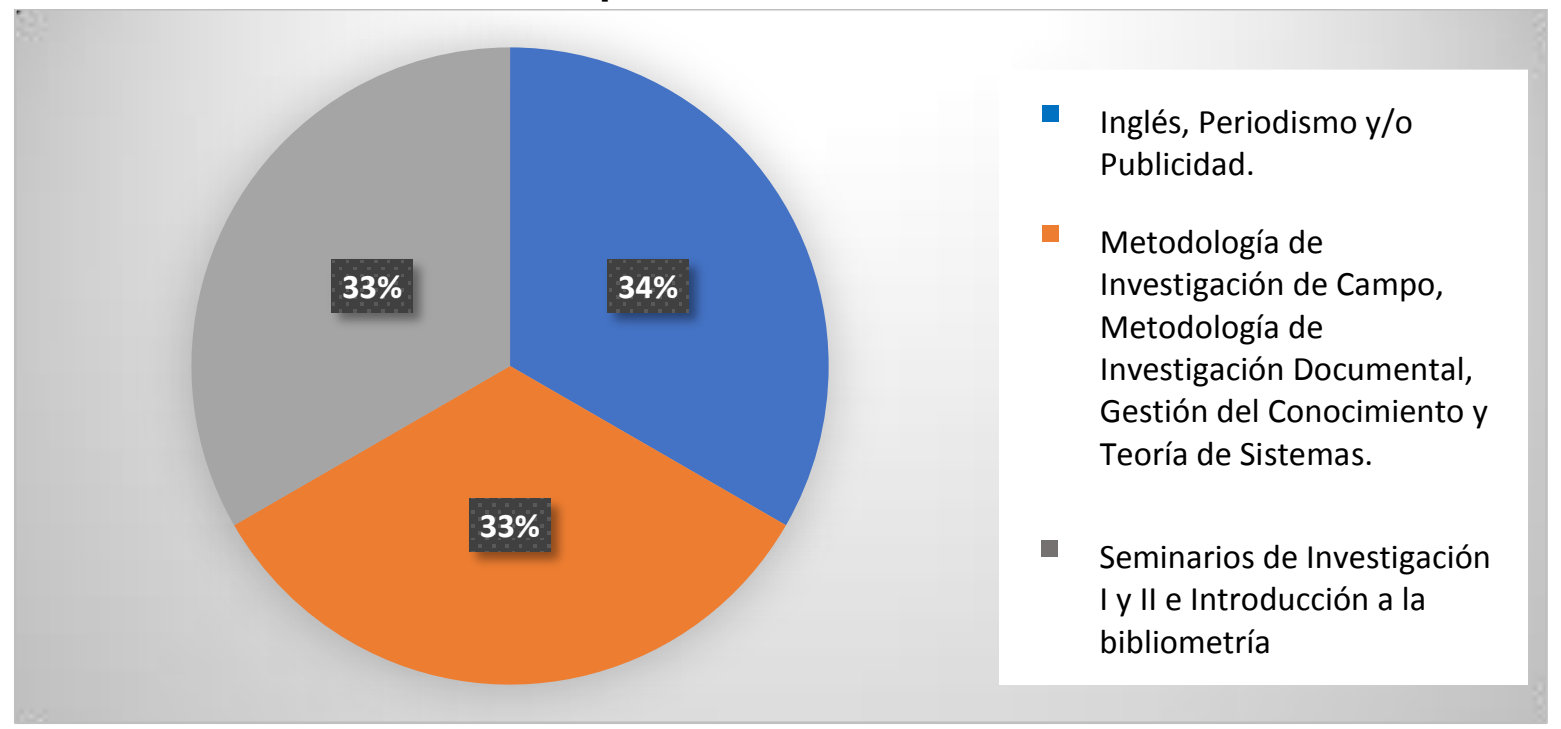

Fuente: elaboración propia de la autora (2017).

Respecto a las actividades adicionales a la docencia se identificó que la mitad de profesores complementa su labor docente haciendo investigación, el resto no lo hace. El 50\% de quienes investigan lo hacen en el área de bibliometría, la otra mitad en metodología de investigación de campo. Mientras que el $100 \%$ de quienes realizan investigación publicaron recientemente. $33 \%$ de los encuestados que ejerce la profesión en una unidad información, además de ejercer la docencia, lo hace en un centro de idiomas, en tanto el $67 \%$ en la biblioteca especializada.

\section{Análisis de los resultados}

Debido a que no todos los docentes enviaron sus respuestas para participar en esta investigación, no se tiene una percepción integral de toda la comunidad; sin embargo, se tiene una muestra representativa ya que en ella participaron profesores de los dos turnos, así como de ambas modalidades; presencial y a distancia. La mayoría de los maestros que enviaron respuesta, se pudo percibir, son quienes tienen una mayor formación profesional. Se aprecia 
en los resultados también que existe mucha participación de los docentes en aspectos relacionados con la investigación, pero esto se debe a que se los solicitan como parte de su carga académica.

También se pudo notar en los resultados que al compararlos con las dimensiones propuestas por la SEP, los docentes se han especializado en los contenidos que imparten, no así, en dominar y estructurar los saberes, ni en facilitar el aprendizaje significativo. Además, pocos maestros planean cada una de sus clases, eso se pudo constatar entrevistando a 30 maestros de la Escuela, en la que mencionaron que casi siempre llevaban presentación de PowerPoint o recomendaban lecturas, pero muy pocos atendían el enfoque por competencias de manera puntual; de la misma forma no ubican los contenidos en los contextos disciplinares, curriculares o sociales.

Por lo cual, al revisar los resultados podemos percibir una ausencia de habilidades y aptitudes de los profesores hacia la práctica didáctica. Y que carecen del perfil docente para lograr la calidad en el proceso de enseñanza y aprendizaje. Y más aún, cuando se revisan las fuentes documentales, se ve que el nuevo perfil del docente es cada día más integral; y que debe tener características muy específicas como las mencionadas por Mas (2012):

De esta manera se requiere del docente universitario con competencia para:

- Conocer, comprender, comunicarse, relacionarse, gestionar y enseñar en y para la diversidad".

- Diseñar la guía docente de acuerdo con las necesidades, el contexto y el perfil profesional, todo ello en coordinación con otros profesionales.

- Desarrollar el proceso de enseñanza-aprendizaje propiciando oportunidades de aprendizaje tanto individual como grupal.

- Tutorizar el proceso de aprendizaje del alumno propiciando acciones que le permitan una mayor autonomía.

- Evaluar el proceso de enseñanza-aprendizaje.

- Contribuir activamente a la mejora de la docencia.

- Participar activamente en la dinámica académico-organizativa de la institución. (p.303). 
Por tanto en el análisis de los resultados se percibe que la gran mayoría de los docentes en la ENBA, aunque tienen los conocimientos sobre el tema que imparten, su perfil carece de las otras 4 dimensiones en las que tendría que ser hábil, apto y con conocimientos para impartir de manera adecuada la materia, así como llevar a cabo propuestas en la planeación en conjunto con la administración de la institución, contribuyendo, en general a la mejora de la práctica didáctica. Así la ENBA tiene varios retos ante sí; trabajar en varias dimensiones y con diferentes actores para lograr lo citado por el autor Mas.

\section{Conclusiones}

Se puede decir que la mayoría de los profesores que respondieron el cuestionario cumplen con el dominio 1 que viene en el perfil docente propuesto por la SEP; ya que en teoría dominan los saberes que transmiten día a día a sus estudiantes. Sin embargo, falta llevar a cabo una adecuada planeación didáctica, ya que es mínima la creación de estrategias encaminadas a lograr el aprendizaje significativo y conseguir que los estudiantes sean seres analíticos y cultos; comprometidos con su formación continua.

También se pudo detectar que los docentes no cubren al 100\% las dimensiones de 2 a la 5 , propuestas por la SEP para el perfil docente; debido a que faltan habilidades y aptitudes para la práctica docente; es decir, falta planificación de los procesos de enseñanza y de aprendizaje, no evalúan, la mayoría de las veces, acordes al modelo educativo, ni al objetivo inicial de la clase. Muchos de ellos no saben ni que es una rúbrica. La mayoría de ellos carece de cursos enfocados a la didáctica en los que les muestren estrategias más efectivas, creativas e innovadoras para llevar a cabo sus cursos.

Se carece, por parte de la Institución Educativa, de estrategias institucionales que permitan que el perfil docente sea congruente con su modelo educativo. Es primordial que se lleven a cabo otros procesos de evaluación para saber si se planifican los procesos de enseñanza y aprendizaje de acuerdo con el modelo educativo de la escuela; así como la manera de evaluar la enseñanza y la transmisión de saberes. 


\section{Referencias}

Asociación Nacional de Universidad e Instituciones de Educación Superior (1986). Glosario de educación superior. México, D.F.: Secretaría de Educación Pública, Asociación Nacional de Universidad e Instituciones de Educación Superior.

Cano, E. (2005). El portafolio del profesorado universitario: un instrumento para la evaluación y el desarrollo profesional. Barcelona: Universitat de Barcelona, Institut de Ciències de l'Educació.

Fernández Borrero, M., \& González Losada, S. (2012). El perfil del buen docente universitario. REDU. Revista de Docencia Universitaria, 10(2), 237-249. DOldoi.org/10.4995/redu.2012.6106

Mas, O. (2012). Las competencias del docente universitario: la percepción del alumno, de los expertos y del propio protagonista.Revista Docencia Universitaria, 10(2), 299-318. Recuperado de https://polipapers.upv.es/index.php/REDU/article/view/6109

México (2013). Programa Sectorial de Educación 2013-2018. Recuperado de http://www.sep.gob.mx/work/models/sep1/Resource/4479/4/images/PROGRAMA SE CTORIAL DE EDUCACION 20132018 WEB.pdf

Sánchez, S. (1995). (Ed.). Diccionario de las ciencias de la educación. México: Santillana.

Secretaría de Educación Pública (2014). Perfil, parámetros e indicadores para el ingreso a las funciones docentes y técnico docentes en la educación media superior. Recuperado dehttp://servicioprofesionaldocente.sep.gob.mx/content/ms/docs/parametros indicadores/Perfi I Parametros Indicadores Docentes.pdf

\section{Notas de la Autora}

* Brenda Cabral Vargas: Instituto de Investigaciones Bibliotecológicas y de la Información, UNAM. Correo electrónico: brendacabralvargas@yahoo.com.mx 